
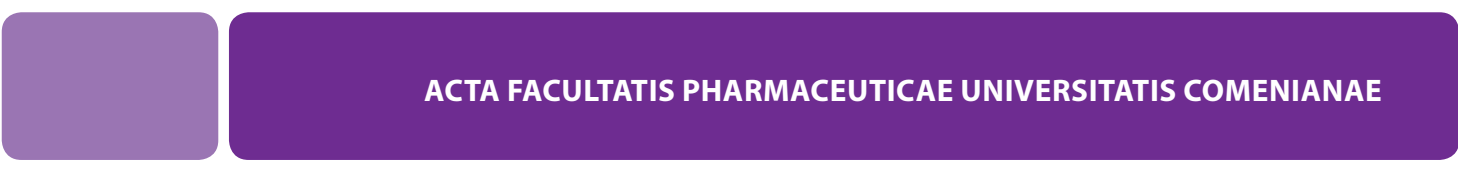

\title{
Evaluation of content of essential oil in selected Salvia L. species Hodnocení obsahu silice ve vybraných druzích rodu Salvia L.
}

${ }^{1}$ Mendel University in Brno, Faculty of Horticulture in Lednice, 691 44, Lednice, Czech Republic ${ }^{2}$ Agricultural Research, Ltd, Troubsko, Czech Republic
${ }^{1}$ Mendelova univerzita v Brně, Zahradnická fakulta,

691 44, Lednice, Česká republika

'Zemědělský výzkum, spol. s r. o. Troubsko, Česká republika

Received November 30, 2014, accepted January 30, 2015

Abstract Salvia L. is multipurpose aromatic plant, used in a wide range of industries, including pharmaceutical, cosmetics, food and above all, in ornamental gardening. The aim of this study is evaluation of essential oil content of ornamental Salvia L. species cultivated in experimental field of Mendel University in Brno, Faculty of Horticulture in Lednice during two periods 2002-2004 and 20092012. Variability of essential oil yield of 42 different taxa was monitored for 9 years. Essential oil content was determined via steam distillation, expressed as $\mathrm{ml} / \mathrm{kg}$. The lowest content for the entire period showed sample S. przewalskii, in year 2010 (content 0.02 $\mathrm{ml} / \mathrm{kg}$ ) and the highest content for the entire period showed sample S. officinalis 'Extracta', in year $2004(22.90 \mathrm{ml} / \mathrm{kg})$. The results of evaluation of essential oil content in Salvia L. are consistent with data in the literature comparing the case of subspecies and varieties of Salvia officinalis L. The average content of essential oil ranges from 6.51 to $19.20 \mathrm{ml} / \mathrm{kg}$.

Slovak Salvia L. je aromatická rostlina, používaná v řadě průmyslových odvětví, včetně farmaceutického, kosmetického, potravinářského abstract a je významná také v okrasném zahradnictví. Cílem této práce je zhodnocení obsahu silice v okrasných taxonech rodu Salvia L., které byly pěstovány na experimentálních plochách Zahradnické fakulty Mendelovy univerzity v Brně, v letech 2002-2004 a 2009-2012. Proměnlivost obsahu silice čtyřiceti dvou různých taxonů byly sledovány po dobu devíti let. Obsah silice byl stanoven pomocí destilace s vodní parou a vyjádřený v $\mathrm{ml} / \mathrm{kg}$. Nejnižší obsah byl, za celé období, shledán ve vzorku S. przewalskii, v roce $2010(0,02 \mathrm{ml} / \mathrm{kg})$ a nejvyšší obsah ve vzorku S. officinalis,Extrakta', v roce $2004(22,90 \mathrm{ml} / \mathrm{kg})$. Výsledky hodnocení obsahu silice v rodu Salvia L. jsou v souladu s údaji uvedenými v literatuře, v př́ípadě porovnání poddruhů a odrůd Salvia officinalis L. Průměrný obsah silice se pohybuje $v$ rozmezí 6,51-19,20 ml/kg.

Keywords sage - Salvia L. - ornamental taxa-essential oil

Klúčové Šalvěj-Salvia L. - okrasné taxony-silice

slová:

\section{INTRODUCTION}

Genus Salvia L., sage, belongs to the family Lamiaceae and included more than 900 species (Štěpánková, 2000). Sage is native to the northeastern Mediterranean region. It is cultivated in Eastern Europe, China, Turkey, Greece, Italy and the United States (Raghavan, 2006). They are mostly annual, biennial or perennial aromatic herbs or semishrub (Štěpánková, 2000). Very branchy and characterised by oblong lanceolate leaves with a glandular surface (lower petiolate leaves, $3-8 \times 3-4 \mathrm{~cm}$; higher leaves smaller and sessile), flowers are clearly bilabiate and falsely whorled in groups of three at the apex of stem. All of the leaves are denticulate, rough to touch and pubescent to both sides (Bruneton, 1999) or rarely glabrate (Štěpánková, 2000). There are many varieties-narrow leaf sage, broad leaf sage, garden sage, tricoloured sage, purple leaf sage, golden sage, red sage, clary sage (France, Russia, and Morocco), Mexican sage, Spanish sage, and pineapple sage. Sage is used fresh or dried, whole, minced, chopped, crushed, rubbed (ground coarsely), or finely ground. Dried sage contains vitamin A, calcium, potassium, niacin, magnesium, phosphorus, and iron. If Salvia used as spice, the taste would depend on the variety and could be from mild to strong camphoric or balsamic. Spanish

*E-mail:neugebj@zf.mendelu.cz

(c) Acta Facultatis Pharmaceuticae Universitatis Comenianae 
sage is less bitter with a flavour in between the flavours of Dalmatian sage and Greek sage. Clary sage has muscat-like notes. Dried sage has a stronger flavour than fresh sage. Sage belongs to spice about strong antioxidant properties (Raghavan, 2006).

Majority of Salvia L. species contain many biological active compounds, but among them, the most important are: essential oil (EO), tannins and bitter compounds (Tekel'ová, 1993). EO is produced in glands and glandular trichomes and it is extracted by steam distillation and has yellowish colour. According to species, there are three kinds of sage EO, which differ by their composition: Dalmatian from Salvia officinalis ssp. minor, Spanish from Salvia lavandulifolia and Greek from Salvia triloba. The most important is Dalmatian sage EO from Salvia officinalis ssp. minor (optionally from ssp. major, which has similar EO like ssp. minor) (Tekelová, 1993). In Germany, only one species is recognised as official, namely, Salvia officinalis subsp. minor (Gmelin) Gams (=S. officinalis sensu stricto) and subsp. major (Garsault) Gams (= S. tomentosa Miller), in contrast S. lavandulifolia is not (Bruneton,1999).

The content and composition of the EO mentioned by particular authors from various parts of the world differs. The differences are due to different climatic and soil conditions and location of the plant. Phenological stage of the plants on the collection date is also very important. In the aerial parts of Salvia officinalis is $0.4-2.5 \%$ EO. The temperature of air plays an important role during plant development. (Tekelová, 1993).

Amr et Đorđević (2000) evaluated EO content of Salvia officinalis from different regions of Jordan and contents ranged between 1.18-2.13\%. The EO yields, expressed in $\mathrm{ml} /$ $\mathrm{kg}$ dried weight (DW), varied from one accession to another according to seasonal collection, ranging from 0.22 to $1.6 \%$. The lowest values were observed in 'Extracta' variety, at fall collection, while the highest result was in 'Regula', at summer collection, during the second year (Menghini et al., 2013). Mirjalili et al. (2006) studied yield of EO (w/w \%) in different stages, and the result is the order: floral budding $(0.9 \%)>$ vegetative $(0.7 \%)>$ flowering $(0.5 \%)>$ immature fruit $(0.4 \%)$ $>$ ripen fruit $(0.2 \%)$.

The quantitative analysis of EO, extracted via hydrodistillation, from different genotypes of Salvia officinalis L. cultivated in Republic of Moldova has been studied. The EO contents of five genotypes of $S$. officinalis are very variable and depend on genotype and collected phase. The highest content of EO was determined in the material collected after flowers and seeds fall: $1.455-1.823 \%$ (dry matter) in shoots with leaves and $1.408-1.749 \%$ (dry matter) in leaves (Gonceariuc et al. 2012). The content of EO in Salvia sclarea is $0.1-0.3 \%$ (Dachler et Pelzmann, 1989).

The influence of year of growing $(2002,2003,2004)$ in the drug quality (Salvia officinalis herba) was observed in warm agro-climatic region of Slovakia by Habán et al. (2007). The average EO content in dry matter, depending on the year, was as follows: $12.7 \mathrm{ml} / \mathrm{kg}$ (2002), $16.00 \mathrm{ml} / \mathrm{kg}$ (2003), $10.10 \mathrm{ml} / \mathrm{kg}$
(2004). The average EO content in each year for varieties was: variety 'Krajová' $12.75 \mathrm{ml} / \mathrm{kg}$ (2002), $16.75 \mathrm{ml} / \mathrm{kg}$ (2003), $10.65 \mathrm{ml} / \mathrm{kg}$ (2004); variety 'Primorska' $12.00 \mathrm{ml} / \mathrm{kg}$ (2002), $15.00 \mathrm{ml} / \mathrm{kg}$ (2003), $8.50 \mathrm{ml} / \mathrm{kg}$ (2004); variety 'Comune' $13.25 \mathrm{ml} / \mathrm{kg}$ (2002), $16.00 \mathrm{ml} / \mathrm{kg}$ (2003), $10.77 \mathrm{ml} / \mathrm{kg}$ (2004).

Several of the world's almost 1000 Salvia L. species have been used in many ways, for example: EO used in perfumery, the flowers used like a rouge, the leaves used for varicose veins, the seed oil as emollient, the roots as tranquiliser (Kintzios, 2000). The qualitative requirements for Salvia are described in pharmacopoeias and norms. If species of genus Salvia L. are used for pharmaceutical industry, they must be evaluated according to minimal qualitative requirement described in Czech Pharmacopoeia 2009. In European part of CP 2009 are described requirements for drugs Salviae officinalis folium a Salviae trilobae folium. And in national part of CP 2009 is chapter about drug Salviae herba-šalvějová nat', which means dried aerial parts of Salvia officinalis L. sensu lato, which must contain minimally $10 \mathrm{ml}$ of EO per $1 \mathrm{~kg}$ of drug (Pharmacopoeia Bohemica 2009). If species of genus Salvia L. are used for food industry, they must be evaluated according to minimal sensory and physico-chemical requirements described in norm ČSN ISO 11165 Šalvěj sušená - Salvia officinalis L. Spice is formed as mixture of dried leaves and tops of inflorescence and that must contain minimally $1.5 \%$ of EO (ml/100g DW) (ČSN ISO 11165). Qualitative requirements for raw material for cosmetic industry are defined by specific inner norms according to processor.

Species of genus Salvia L. are very valuable ornamental plants because they have very broad spectrum of interesting visual characteristics. The morphology is very variable, from low species (for example, S. jurisicii, S. taraxacifolia) to high (S. glutinosa). Decorative function is also based on colour of species and cultivars, some of them are interesting for shape and colour of leaves, from light green (S. przewalskii) over grey green (S. argentea) to variegated (S. officinalis 'Icterina'), and also the colour and shape of flowers (S. hians, S. pratensis) and bracts (S. sclarea). Landscape uses are very variable, because species of genus Salvia L. could be used in perennial borders, from xeric (S. sclarea) to moist (S. tomentosa), but there are species tolerant to both (S. transsylvanica), also for rock gardens (S. taraxacifolia, S. jurisicii), for herb gardens (S. officinalis, S. sclarea), or could be used like for edging pathways (S. officinalis), as a bee and butterfly plant (S. pratensis) or for cultivating in pots (S. viridis, S. splendens) (Whittlesey, 2014). Clebsch (2008) recommends following species of Salvia for hot and humid climate: S. coccinea and S. splendens.

\section{MATERIALS AND METHODS}

\section{Materials}

During two periods (2002-2004 and 2009-2012) were evaluated 76 samples (Table 1), taxa are named according 
Table 1 List of Salvia L. taxa

\begin{tabular}{|c|c|c|c|}
\hline No. & Species/variety & Supplier & Planting date \\
\hline 1 & S. amplexicaulis & Jelitto, Germany & 16.5.2002 \\
\hline 2 & S. argentea & Jelitto, Germany & 16.5.2002 \\
\hline 3 & S. aethiopis & ZF MENDELU, Czech Republic & 16.5.2002 \\
\hline 4 & S. hians & Jelitto, Germany & 16.5.2002 \\
\hline 5 & S. jurisicii & Jelitto, Germany & 16.5.2002 \\
\hline 6 & S. nemorosa & Jelitto, Germany & 16.5.2002 \\
\hline 7 & S. nemorosa 'Rosenwein' & Jelitto, Germany & 16.5.2002 \\
\hline 8 & S. officinalis f. albiflora & Jelitto, Germany & 16.5.2002 \\
\hline 9 & S. officinalis 'Rosea' & Jelitto, Germany & 16.5.2002 \\
\hline 10 & S. officinalis & LF MU, Czech Republic & 16.5.2002 \\
\hline 11 & S. pratensis & Jelitto, Germany & 16.5.2002 \\
\hline 12 & S. sclarea & Jelitto, Germany & 16.5.2002 \\
\hline 13 & S. transsylvanica & Jelitto, Germany & 16.5.2002 \\
\hline 14 & S. verticillata & Jelitto, Germany & 16.5.2002 \\
\hline 15 & S. verticillata 'Alba' & Jelitto, Germany & 16.5.2002 \\
\hline 16 & S. $x$ superba 'Blaukönigin' & Jelitto, Germany & 16.5.2002 \\
\hline 17 & S. x superba 'Rosakönigin' & Jelitto, Německo & 16.5.2002 \\
\hline 18 & S. officinalis 'Icterina' & ZF MENDELU, Czech Republic & 16.5.2002 \\
\hline 19 & S. officinalis 'Purpurescens' & ZF MENDELU, Czech Republic & 16.5.2002 \\
\hline 20 & $\begin{array}{l}\text { S. pratensis subsp. haematodes } \\
\text { 'Mittsommer' }\end{array}$ & Jelitto, Germany & 25.5 .2003 \\
\hline 21 & S. przewalskii & Jelitto, Germany & 25.5 .2003 \\
\hline 22 & S. aethiopis & ZF MENDELU, Czech Republic & 25.5.2003 \\
\hline 23 & S. bulleyana & Jelitto, Germany & 25.5.2003 \\
\hline 24 & S. ringens & Jelitto, Germany & 25.5.2003 \\
\hline 25 & S. sclarea 'Vatican White' & Jelitto, Germany & 25.5.2003 \\
\hline 26 & S. nemorosa var. tesquicola & Jelitto, Germany & 25.5.2003 \\
\hline 27 & S. officinalis 'Extrakta' & Jelitto, Germany & 25.5.2003 \\
\hline 28 & S. nemorosa 'Rosakönigin' & BG Grugapark Essen, Germany & 22.5.2009 \\
\hline 29 & S. verticillata & BG Wroclaw, Poland & 22.5.2009 \\
\hline 30 & S. officinalis 'Rosea' & LF MU, Czech Republic & 22.5.2009 \\
\hline 31 & S. aethiopis & LF MU, Czech Republic & 2.6 .2009 \\
\hline 32 & S. transylvanica & LF MU, Czech Republic & 2.6.2009 \\
\hline 33 & S. argentea & LF MU, Czech Republic & 2.6.2009 \\
\hline 34 & S. afficinalis 'Alba' & LF MU, Czech Republic & 22.5.2009 \\
\hline 35 & S. nemorosa ssp. tesquicola & LF MU, Czech Republic & 2.6.2009 \\
\hline 36 & S. sclarea & Bundesgärten Wien, Austria & 22.5.2009 \\
\hline 37 & S. argentea & Bundesgärten Wien, Austria & 22.5.2009 \\
\hline 38 & S. glutinosa & BG Univerzita Salzburg, Austria & 22.5.2009 \\
\hline
\end{tabular}


Evaluation of content of essential oil in selected Salvia L. species

Continued Table 1. List of Salvia L. taxa

\begin{tabular}{|c|c|c|c|}
\hline No. & Species/variety & Supplier & Planting date \\
\hline 39 & S. officinallis & BG Wroclaw, Poland & 22.5 .2009 \\
\hline 40 & S. fruticosa & BG Wroclaw, Poland & 22.5.2009 \\
\hline 41 & S. prewalskii & BG Wroclaw, Poland & 22.5.2009 \\
\hline 43 & S. sclarea & BG Wroclaw, Poland & 22.5.2009 \\
\hline 44 & S. nemorosa & BG Wroclaw, Poland & 22.5.2009 \\
\hline 45 & S. tillifolia & BG Wroclaw, Poland & 22.5.2009 \\
\hline 46 & S. verticillata & BG Wroclaw, Poland & 2.6.2009 \\
\hline 47 & S. nemorosa ssp. tesquicola & Jelitto, Germany & 22.5 .2009 \\
\hline 48 & S. nemorosa 'Rosenwein' & Jelitto, Germany & 22.5.2009 \\
\hline 49 & S. nemorosa 'Blaukönigin' & Jelitto, Germany & 22.5.2009 \\
\hline 50 & S. nemorosa 'Rosakönigin' & Jelitto, Germany & 2.6.2009 \\
\hline 51 & S. nemorosa 'Violetkönigin' & Jelitto, Germany & 22.5 .2009 \\
\hline 52 & S. sclarea & Jelitto, Germany & 22.5.2009 \\
\hline 53 & S. officinalis 'Extrakta' & Jelitto, Germany & 22.5.2009 \\
\hline 54 & S. jurisicii & Jelitto, Germany & 2.6.2009 \\
\hline 55 & S. amplexicaulis & Jelitto, Germany & 22.5.2009 \\
\hline 56 & S. hians & Jelitto, Germany & 22.5 .2009 \\
\hline 57 & S. pratensis & Jelitto, Germany & 22.5.2009 \\
\hline 58 & S. sclarea 'Kardinal' & BG Kiev, Ukraine & 22.5.2009 \\
\hline 59 & S. aethiopis & BG Kiev, Ukraine & 22.5.2009 \\
\hline 60 & S. bicolor & BG Kiev, Ukraine & 22.5.2009 \\
\hline 61 & S. reflexa & BG Kiev, Ukraine & 22.5.2009 \\
\hline 62 & S. virgata & BG Kiev, Ukraine & 22.5.2009 \\
\hline 63 & S. splendens & BG Kiev, Ukraine & 22.5 .2009 \\
\hline 64 & S. coccinea & BG Kiev, Ukraine & 22.5 .2009 \\
\hline 65 & S. triloba & BG Kiev, Ukraine & 22.5 .2009 \\
\hline 66 & S. verticilata & BG VILAR, Moscow, Russia & 2.6 .2009 \\
\hline 67 & S. deserta & BG Bishkek, Kyrgystan & 2.6.2009 \\
\hline 68 & S. hispanica & $*$ & 22.6.2009 \\
\hline 69 & S. miltiorrhiza & Martinkov, Czech Republic & 20.6.2010 \\
\hline 70 & S. tillifolia & BG Wroclaw, Poland & 1.6.2010 \\
\hline 71 & S. reflexa & BG Kiev, Ukraine & 1.6.2010 \\
\hline 72 & S. coccinea & BG Kiev, Ukraine & 1.6.2010 \\
\hline 73 & S. sclarea 'Vatican White' & ZF MENDELU, Czech Republic & 1.6.2010 \\
\hline 74 & S. officinalis & VúPT Troubsko, Czech Republic & 15.5.2010 \\
\hline 75 & S. officinalis lavandulifolia & Bundesgärten Wien, Austria & 2.8 .2010 \\
\hline 76 & S. nemorosa & Bundesgärten Wien, Austria & 2.8 .2010 \\
\hline \multicolumn{2}{|c|}{ BG botanical garden,*unidentified } & & \\
\hline
\end{tabular}


Table 2. The average amount of hours of sunshine $[\mathrm{h}]$ and air temperature $\left[{ }^{\circ} \mathrm{C}\right]$

\begin{tabular}{|c|c|c|c|c|c|c|c|c|c|c|}
\hline \multirow{2}{*}{ year } & \multicolumn{2}{|c|}{ April } & \multicolumn{2}{c|}{ May } & \multicolumn{2}{c|}{ June } & \multicolumn{2}{c|}{ July } & \multicolumn{2}{c|}{ August } \\
\cline { 2 - 11 } & {$[\mathbf{h}]$} & {$\left[{ }^{\circ} \mathbf{C}\right]$} & {$[\mathbf{h}]$} & {$\left[{ }^{\circ} \mathbf{C}\right]$} & {$[\mathbf{h}]$} & {$\left[{ }^{\circ} \mathbf{C}\right]$} & {$[\mathbf{h}]$} & {$\left[{ }^{\circ} \mathbf{C}\right]$} & {$[\mathbf{h}]$} & {$\left[{ }^{\circ} \mathbf{C}\right]$} \\
\hline 2002 & 150.8 & 9.9 & 208.0 & 17.5 & 259.5 & 19.5 & 352.2 & 21.4 & 187.6 & 20.1 \\
\hline 2003 & 192.8 & 9.7 & 267.4 & 17.6 & 284.9 & 21.7 & 244.9 & 20.7 & 22.9 & 22.9 \\
\hline 2004 & 184.1 & 11.5 & 214.2 & 13.7 & 195.8 & 17.6 & 210.6 & 19.7 & 248.9 & 20.3 \\
\hline 2009 & 262.9 & 14.6 & 227.4 & 16.7 & 177.9 & 17.9 & 283.2 & 21.1 & 265.7 & 20.8 \\
\hline 2010 & 225.9 & 10.7 & 105.8 & 14.5 & 130.3 & 19.0 & 280.4 & 22.3 & 229.5 & 19.7 \\
\hline 2011 & 202.3 & 12.7 & 269.9 & 15.2 & 242.1 & 19.7 & 280.4 & 22.3 & 203.0 & 19.5 \\
\hline 2012 & 213.9 & 11.1 & 269.2 & 16.8 & 221.7 & 19.8 & 215.6 & 20.9 & 273.0 & 20.5 \\
\hline
\end{tabular}

to supplier of plants. Collection of Salvia L. genus was found in the year 2002 and has been enriched by new species until now. The highest number of varieties in collection is varieties of three species: Salvia nemorosa L., Salvia officinalis L. and Salvia $\times$ superba Stapf.

Plants of Salvia L. taxa were cultivated in experimental field of Mendel University in Brno, Faculty of Horticulture in Lednice ( $48^{\circ} 48^{\prime} \mathrm{N} 16^{\circ} 48^{\prime} \mathrm{E}$, altitude $176 \mathrm{~m}$ ) in the south of Moravia, Czech Republic. The climatic conditions of the Lednice area during 2002-2004 and 2009-2012 are summarised in Table 2 (Vachůn, 2014).

Aerial parts of all taxa were harvested manually at stage of full flowering (means May-July), then naturally dried and stored in paper bags until next analysis.

\section{Quantitative analysis of EO}

The EO of the dry flowering aerial parts was extracted by steam distillation according to Pharmacopoeia Bohemica (2009), modified procedure, without xylene. Samples of dried herb were fragmented in a laboratory mill (ILABO MF 10 basic, maximally size of grain $3.15 \mathrm{~mm}$ ) maximally 24 hour before distillation. Plant material $(10 \mathrm{~g})$ was distilled in $500 \mathrm{ml} \mathrm{DH}_{2} \mathrm{O}$ in a $1000 \mathrm{ml}$ flask for 90 minutes. All samples were analysed duplicate and averaged. EO content is expressed as $\mathrm{ml} / \mathrm{kg}$.

\section{RESULTS}

The average of EO content of all the 42 taxa during 20022004 and 2009-2012 was following: S. aethiopis $3.01 \mathrm{ml} / \mathrm{kg}$, S. amplexicaulis $1.76 \mathrm{ml} / \mathrm{kg}$, S. argentea $2.46 \mathrm{ml} / \mathrm{kg}$, S. bicolor $0.84 \mathrm{ml} / \mathrm{kg}$, S. bulleyana $5.30 \mathrm{ml} / \mathrm{kg}$, S. coccinea $0.54 \mathrm{ml} / \mathrm{kg}$, S. deserta $0.66 \mathrm{ml} / \mathrm{kg}$, S. fruticosa $8.42 \mathrm{ml} / \mathrm{kg}$, S. glutinosa $1.52 \mathrm{ml} / \mathrm{kg}$, S. hians $1.83 \mathrm{ml} / \mathrm{kg}$, S. hispanica $0.81 \mathrm{ml} / \mathrm{kg}$, S. jurisicii $2.51 \mathrm{ml} / \mathrm{kg}$, S. miltiorrhiza $0.74 \mathrm{ml} / \mathrm{kg}, \quad$ S. nemorosa 'Violetkönigin' $1.34 \mathrm{ml} / \mathrm{kg}$, S. nemorosa'Rosenwein' $2.62 \mathrm{ml} / \mathrm{kg}$, S. nemorosa var. tesquicola $2.19 \mathrm{ml} / \mathrm{kg}$, S. nemorosa $2.09 \mathrm{ml} / \mathrm{kg}$, S. officinalis 'Extrakta' $10.16 \mathrm{ml} / \mathrm{kg}$, S. officinalis 'Icterina' $19.20 \mathrm{ml} / \mathrm{kg}$, S. officinalis 'Purpurescens' $6.51 \mathrm{ml} / \mathrm{kg}$, S. officinalis 'Rosea' $12.20 \mathrm{ml} / \mathrm{kg}$, S. officinalis f. albiflora $9.30 \mathrm{ml} / \mathrm{kg}$, S. officinalis ssp. lavandulifolia $7.71 \mathrm{ml} / \mathrm{kg}$, S. officinalis $9.52 \mathrm{ml} / \mathrm{kg}$, S. pratensis subsp. haematodes
'Mittsommer' $3.06 \mathrm{ml} / \mathrm{kg}$, S. pratensis $3.36 \mathrm{ml} / \mathrm{kg}$, S. przewalskii $2.84 \mathrm{ml} / \mathrm{kg}$, S. reflexa $1.08 \mathrm{ml} / \mathrm{kg}$, S. ringens $9.03 \mathrm{ml} / \mathrm{kg}$, S. sclarea 'Kardinal' $1.07 \mathrm{ml} / \mathrm{kg}$, S. sclarea 'Vatikan White' $3.80 \mathrm{ml} / \mathrm{kg}$, S. sclarea $4.14 \mathrm{ml} / \mathrm{kg}$, S. splendens $1.81 \mathrm{ml} / \mathrm{kg}$, S. $\times$ superba 'Blaukönigin' $2.02 \mathrm{ml} / \mathrm{kg}$, S. $\times$ superba'Rosakönigin' $2.23 \mathrm{ml} / \mathrm{kg}$, S. tillifolia $0.97 \mathrm{ml} / \mathrm{kg}$, S. transsylvanica $1.86 \mathrm{ml} / \mathrm{kg}$, S. triloba $6.13 \mathrm{ml} / \mathrm{kg}$, S. verbenaca $1.08 \mathrm{ml} / \mathrm{kg}$, S. verticillata 'Alba' $4.21 \mathrm{ml} / \mathrm{kg}$, S. verticillata $1.43 \mathrm{ml} / \mathrm{kg}$, S. virgata $0.89 \mathrm{ml} / \mathrm{kg}$.

Some of the taxa were not included in the overall survey results (Table 3), because the content of EO was evaluated in only one year.

The Table 3 and Figure 1 represented only taxa that were evaluated at least 2 years during two periods 2002-2004 and 2009-2012.

EO content in year 2002 ranged between 0.40 (14S. verticillata; 17 S. $\times$ superba 'Rosakönigin')-1.00 (1 S. amplexicaulis; 5 S. jurisicii; 6 S. nemorosa; 7 S. nemorosa 'Rosenwein') ml/ $\mathrm{kg}$, in year 2003 ranged 0.40 (14 S. verticillata)-10.65 (10 S. officinalis) $\mathrm{ml} / \mathrm{kg}$, in year 2004 ranged between 6.90 (20 S. pratensis subsp. haematodes 'Mittsommer')-22.90 (27 S. officinalis 'Extrakta') $\mathrm{ml} / \mathrm{kg}$, in year 2009 ranged between 0.54 (29 S. verticillata)-4.14 (19 S. officinalis 'Purpurescens') $\mathrm{ml} / \mathrm{kg}$, in year 2010 ranged between 0.02 (41 S. przewalskii)-10.45 (39 S. officinalis) $\mathrm{ml} / \mathrm{kg}$, in year 2011 ranged between 0.36 (55 S. amplexicaulis)-11.06 (40 S. fruticosa) $\mathrm{ml} / \mathrm{kg}$, and finally, in year 2012 ranged between 0.36 (32 S. transsylvanica)-8.46 (65 S. triloba) $\mathrm{ml} / \mathrm{kg}$.

We can say, according to above-mentioned contents of EO, that during the entire period 2002-2004 and 2009-2012, the lowest content showed in year 2010, with content being $0.02 \mathrm{ml} / \mathrm{kg}$ (41 S. przewalskii) and the highest content $22.90 \mathrm{ml} / \mathrm{kg}$, in year 2004 (27 S. officinalis 'Extracta').

\section{DISCUSSION}

The results of evaluation of EO content in Salvia L. are consistent with data in the literature comparing subspecies and varieties of Salvia officinalis $\mathrm{L}$. The average content of EO ranges from 6.51 to $19.20 \mathrm{ml} / \mathrm{kg}$ (Tekelová 1993, Amr et Đorđević 2000, Gonceariuc et al. 2012).

These samples (sample, $\mathrm{ml} / \mathrm{kg}$, year): 10 S. officinalis $10.65 \mathrm{ml} / \mathrm{kg}$ in 2003 and $10.45 \mathrm{ml} / \mathrm{kg}$ in 2010; 27 S. officinalis 'Extracta' 
Evaluation of content of essential oil in selected Salvia L. species

Table 3. Essential oil content of analysed samples Salvia L. [m/ $/ \mathrm{kg}]$

\begin{tabular}{|c|c|c|c|c|c|c|c|}
\hline No. & 2002 & 2003 & 2004 & 2009 & 2010 & 2011 & 2012 \\
\hline 1 & 1.00 & 0.45 & 7.25 & $*$ & $*$ & * & $*$ \\
\hline 5 & 1.00 & 0.95 & 8.45 & * & * & $*$ & * \\
\hline 6 & 1.00 & 0.80 & 9.35 & * & * & * & * \\
\hline 7 & 1.00 & 1.25 & 9.13 & $*$ & * & * & * \\
\hline 8 & $*$ & 5.20 & 13.39 & $*$ & $*$ & $*$ & $*$ \\
\hline 9 & $*$ & 6.80 & 22.25 & $*$ & $*$ & $*$ & $*$ \\
\hline 10 & * & 10.65 & 9.35 & $*$ & $*$ & $*$ & $*$ \\
\hline 13 & 0.70 & 0.60 & 7.18 & * & * & * & $*$ \\
\hline 14 & 0.40 & 0.40 & 7.68 & * & * & * & * \\
\hline 15 & 0.60 & 0.60 & 7.45 & * & * & * & * \\
\hline 16 & 0.60 & 0.60 & 8.88 & * & * & * & * \\
\hline 17 & 0.40 & 0.60 & 9.00 & * & * & * & * \\
\hline 19 & $*$ & ${ }^{*}$ & 8.85 & 4.16 & ${ }^{*}$ & ${ }^{*}$ & ${ }^{*}$ \\
\hline 20 & * & $*$ & 6.90 & 2.01 & 0.27 & * & * \\
\hline 21 & * & * & 10.15 & 1.08 & 0.81 & 1.67 & $*$ \\
\hline 25 & ${ }^{*}$ & * & 10.15 & 1.21 & ${ }^{*}$ & ${ }^{*}$ & $*$ \\
\hline 27 & * & ${ }^{*}$ & 22.9 & $*$ & * & ${ }^{*}$ & 3.70 \\
\hline 28 & * & * & ${ }^{*}$ & 1.23 & 1.64 & 0.99 & * \\
\hline 29 & * & * & * & 0.54 & 0.45 & 1.45 & * \\
\hline 32 & * & * & * & 1.25 & 0.74 & 0.67 & 0.36 \\
\hline 33 & * & * & * & ${ }^{*}$ & 1.09 & 1.00 & * \\
\hline 35 & * & ${ }^{*}$ & * & 0.80 & 0.40 & 0.98 & 0.54 \\
\hline 37 & * & ${ }^{*}$ & * & ${ }^{*}$ & 0.95 & 1.11 & $*$ \\
\hline 39 & * & ${ }^{*}$ & ${ }^{*}$ & ${ }^{*}$ & 10.45 & 9.83 & 7.09 \\
\hline 40 & * & * & $*$ & $*$ & 9.82 & 11.06 & 4.39 \\
\hline 41 & * & * & * & & 0.02 & 3.32 & $*$ \\
\hline 44 & ${ }^{*}$ & ${ }^{*}$ & ${ }^{*}$ & * & 0.43 & 0.88 & ${ }^{*}$ \\
\hline 46 & * & ${ }^{*}$ & ${ }^{*}$ & 1.68 & 0.55 & 1.13 & ${ }^{*}$ \\
\hline 47 & * & ${ }^{*}$ & * & 0.80 & 0.54 & 0.45 & * \\
\hline 48 & * & * & * & 1.03 & 1.08 & 2.20 & * \\
\hline 49 & $*$ & $*$ & ${ }^{*}$ & 0.67 & 0.36 & 1.01 & * \\
\hline 51 & ${ }^{*}$ & * & * & 0.80 & 2.87 & 0.55 & 1.12 \\
\hline 53 & * & * & * & * & 10.08 & 10.21 & 3.90 \\
\hline 54 & * & ${ }^{*}$ & * & 1.29 & 0.88 & * & * \\
\hline 55 & ${ }^{*}$ & ${ }^{*}$ & * & 0.81 & 0.68 & 0.36 & * \\
\hline 56 & $*$ & * & $*$ & $*$ & 0.80 & 1.00 & $*$ \\
\hline 57 & * & * & * & 2.03 & ${ }^{*}$ & $*$ & 0.37 \\
\hline 59 & ${ }^{*}$ & ${ }^{*}$ & ${ }^{*}$ & * & 0.90 & 2.51 & ${ }^{*}$ \\
\hline 62 & ${ }^{*}$ & ${ }^{*}$ & ${ }^{*}$ & ${ }^{*}$ & 1.00 & 0.77 & * \\
\hline 65 & * & * & * & * & 3.90 & * & 8.46 \\
\hline 66 & ${ }^{*}$ & * & * & 0.80 & ${ }^{*}$ & 0.67 & $*$ \\
\hline 73 & * & * & $*$ & $*$ & $*$ & 2.71 & 1.11 \\
\hline
\end{tabular}




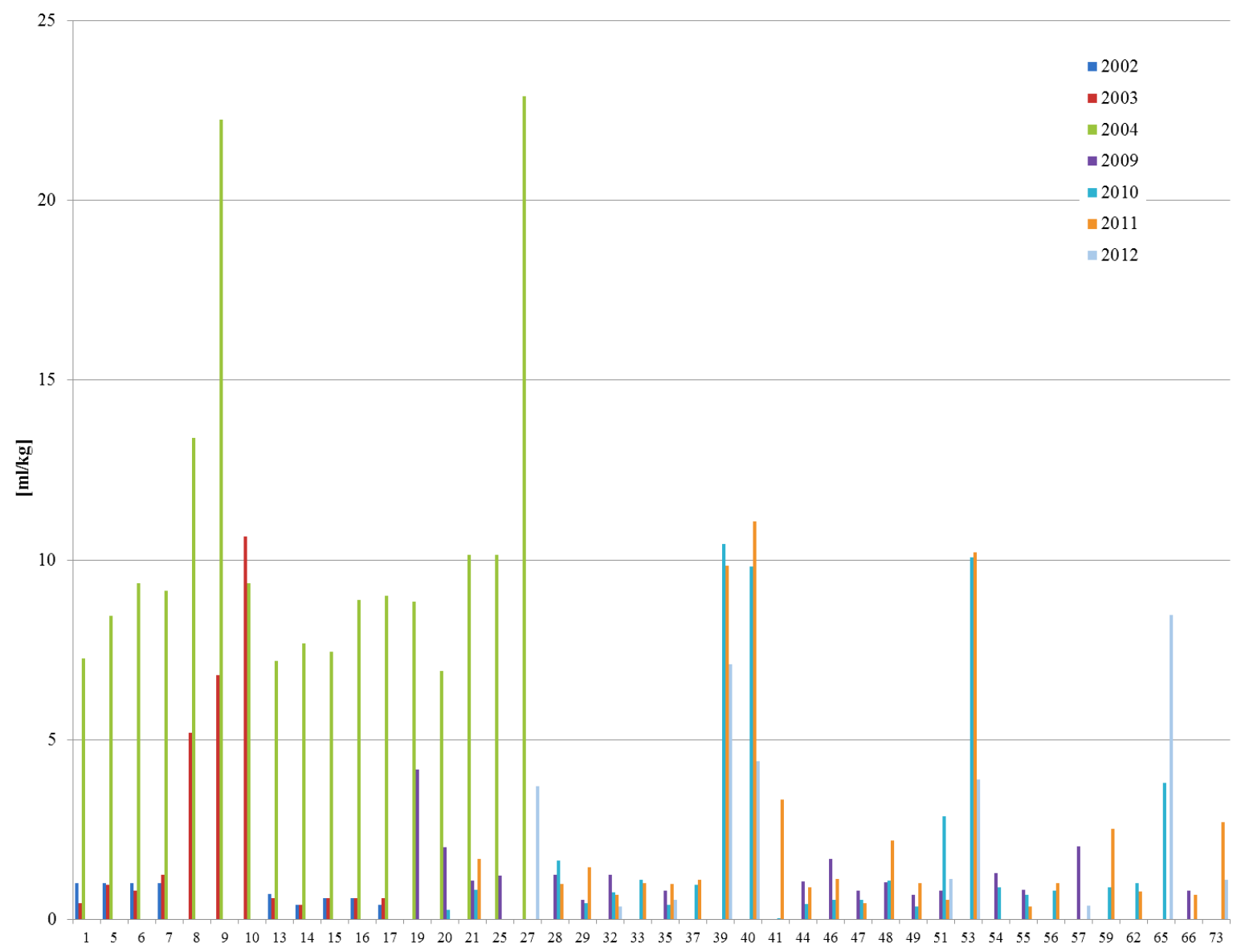

Figure 1. Graph of essential oil content [ $\mathrm{ml} / \mathrm{kg}]$ EO: essential oil

$22.90 \mathrm{ml} / \mathrm{kg}$ in 2004 and $10.08 \mathrm{ml} / \mathrm{kg}$ in 2010 and $10.21 \mathrm{ml} / \mathrm{kg}$ in 2011; 18 S. officinalis 'Icterina' $19.20 \mathrm{ml} / \mathrm{kg}$ in 2004; 9 S. officinalis 'Rosea' $22.25 \mathrm{ml} / \mathrm{kg}$ in 2004; $8 \mathrm{~S}$. officinalis $\mathrm{f}$. albiflora $13.39 \mathrm{ml} / \mathrm{kg}$ in 2004, met with minimum requirement for EO content described in Czech Pharmacopoeia 2009.

These samples (sample, $\mathrm{ml} / \mathrm{kg}$, year): 27 S. officinalis 'Extracta' $22.90 \mathrm{ml} / \mathrm{kg}$ in 2004; $18 \mathrm{~S}$. officinalis 'Icterina' $19.20 \mathrm{ml} / \mathrm{kg}$ in 2004; 9 S. officinalis 'Rosea' 22.25 ml/kg in 2004, met with minimum requirement for EO content described norm ČSN ISO 11165.

If we compare information about EO content of $S$. officinalis cultivated in similar agroclimatic areas, while average contents ranged between $8.50-16.00 \mathrm{ml} / \mathrm{kg}$, we found match with our results, because our average EO contents ranged between $6.51-19.20 \mathrm{ml} / \mathrm{kg}$. Our average EO contents observed in 2004 were higher than in 2003, while Habán et al. (2007) reached higher contents in 2003 than 2004, which is interesting, because the weather condition in 2003 was better for producing EO than 2004 (means higher average of temperature and hours of sunshine), see Table 2. Extreme differences between EO content in each year may be due to storage period and/or storage conditions. EO content can also be influenced by ratio of leaves:flowers:stems in samples, but in this case, this ratio has not been evaluated.
Average EO content of S. sclarea was higher (1.63-9.60 ml/kg) than values listed by Dachler et Pelzmann (1989).

Information about EO content of ornamental species of sage are not listed in literature focussed on ornamental species of Salvia L. (Clebsch 2008, Whittlesey 2014).

\section{CONCLUSIONS}

Salvia officinalis L. (means also subspecies and variety), which is important in ornamental gardening and is used mainly for its colourful leaves, met minimum qualitative requirements for essential oil content for both norm (Czech Pharmacopoeia 2009 and ČSN ISO 11165). But it is unacceptable to use subspecies and variety with coloured and variegated leaves for pharmaceutical and food industry. We can say there is a lack of information about EO content of ornamental sage species in literature.

\section{AKNOWLEDGEMENT}

Thisstudywassupported bygrantOPVKCZ.1.07/2.4.00/31.0089 of the Ministry of Education of the Czech Republic. 
[1] Bruneton J. Pharmacognosy, Phytochemistry Medicinal Plants, 1st ed., Paris Lovoisier Publishing; 1999.

[2] Clebsch B. The new book of salvias: sages for every garden. 2nd ed. Portland, Or: Timber; 2008.

[3] Kintzios SE. Sage. The Genus Salvia. 1st ed. Amsterdam, Harwood Academic Publishers; 2000.

[4] Pharmacopoeia Bohemica 2009, Grada Publishing Praha; 2009.

[5] Raghavan S. Spices, Seasonings and Flavorings. London, Taylor\&Francis Group; 2006.

[6] Štěpánková J. Salvia L. In Slavík B. Květena české republiky (6. díl), 1. vyd., Praha, Academia; 2000.

[7] Tekel'ová D. Salvia officinalis L., Botanická charakteristika, obsahové látky, použitie, pestovanie. Československá farmacie. 1993;XLII/3:111-116.

[8] Whittlesey J. The plant lover's guide to salvias. 1st ed. London,Timber Press; 2014.

[9] Amr S, Đorđević S. The investigation of the quality of sage (Salvia officinalis L.) originating from Jordan. FACTA Universitatis Series, Niš. 2000;5:103-108.

[10] Menghini LL, Pintore G, Chessa M, Tirillini B. Essential oil content and composition of three sage varieties grown in Central Italy. J. Med. Plants Res. 2013;7/9:480-489.

[11] Mirjalili MH, Salehi P, Sonboli A, Vala MM. Essential oil variation of Salvia officinalis aerial parts during its phonological cycle. Chem. Nat. Compd. 2006;42/1:19-23.
[12] ČSN ISO 11165 - šalvěj sušená (Salvia officinalis L.)

[13] Gonceariuc M, Kulcitki V, Balmush Z, Sirbu T, Gonceariuc N, Cuzmin E. Genotypes of Salvia officinalis L. with different essential oil content and composition. [CD-ROM]. In Proceedings of the Seventh Conference on Medicinal and Aromatics Plants of Southeast European Countries. Belgrade, Institute for Medicinal Plants Research "Dr Josif Pančić" Belgrade and AMAPSEEC. 2012;397-401.

[14] Dachler M, Pelzmann H. Heil-und Gewürzpflanzen. Wien, Österreichischer Agrarverlag; 1989.

[15] Habán M, Otepka P, Souikat H, Bokor P, Poláček M. Influence of agri-ecological conditions on yield and essential oil content in sage (Salvia officinalis L.). In Habán, M.; Otepka, P. (ed.) Book of Scientific Papers and Abstracts: 1st International Scientific Conference on Medicinal, Aromatic and Spice Plants, December 5 -- 6, 2007, Slovak University of Agriculture in Nitra, Slovak Republic. SPU, Nitra: Slovak University of Agriculture in Nitra, Slovak Republic. 2007;209.

[16] Vachůn M. Meteorologická stanice ČHMU (http://tilia.zf.mendelu. cz/ustavy/571/meteo_aktual.html). Revised May 6, 2011. Accessed Juni 26, 2014. 